\title{
Antipsychotics and Lorazepam During Delirium: Are We Harming Older Patients? A Real-Life Data Study
}

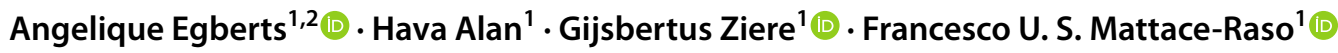

Accepted: 24 October 2020 / Published online: 9 November 2020

(c) The Author(s) 2020

\begin{abstract}
Background Delirium affects approximately one out of three older hospitalized patients and is associated with poor clinical outcomes. Approaches used to manage delirium consist of non-pharmacological and pharmacological interventions. Antipsychotics and lorazepam are commonly used to treat symptoms of delirium, but conflicting data exist on the effect of these drugs on the outcomes of delirium.

Objective The aim of this study was to investigate whether the use of antipsychotics, with or without lorazepam, increases the risk of prolonged hospital stay, post-discharge institutionalization, and in-hospital mortality in older patients with delirium. Methods In this retrospective chart review study, we included acutely ill patients aged $\geq 65$ years who were admitted to a geriatric ward and diagnosed with delirium. Patients were stratified into three groups based on whether or not they received antipsychotics and lorazepam to manage delirium: (0) no antipsychotics; (1) antipsychotics only; and (2) antipsychotics plus lorazepam. Length of hospital stay (LOS) and frequencies of post-discharge institutionalization and in-hospital mortality were compared.

Results In total, 212 patients with delirium were included (mean age $81.9 \pm 5.6$ years); 40 did not receive antipsychotics (18.9\%), 123 received antipsychotics only (58.0\%) and 49 received antipsychotics and lorazepam (23.1\%). There was a trend to a longer LOS in patients who received both antipsychotics and lorazepam (median LOS group $0=8.0$ days, group $1=$ 10.0 days, and group $2=12.0$ days). Furthermore, trends to a higher incidence of post-discharge institutionalization and in-hospital mortality were observed in patients who received both treatments (institutionalization group $0=45.0 \%$, group 1 $=59.3 \%$, group $2=81.6 \%$; and in-hospital mortality group $0=7.5 \%$, group $1=10.6 \%$, group $2=16.3 \%$ ).

Conclusion The use of antipsychotics, with or without lorazepam, during delirium is associated with increased risks of poor outcomes. These findings suggest that clinicians should be cautious about routine prescribing of these drugs to older patients with delirium. Further investigation is needed to clarify this association.
\end{abstract}

\section{Introduction}

Delirium is a serious problem, affecting approximately one out of three older hospitalized persons, and is associated with poor outcomes, including prolonged hospital stay and increased rates of post-discharge institutionalization and cognitive decline $[1,2]$. One-quarter of patients

Angelique Egberts

a.egberts@erasmusmc.nl

1 Section of Geriatric Medicine, Department of Internal Medicine, Erasmus MC University Medical Center, Room Rg-527, PO Box 2040, 3000 CA Rotterdam, The Netherlands

2 Department of Hospital Pharmacy, Franciscus Gasthuis and Vlietland, Rotterdam and Schiedam, The Netherlands who have experienced delirium die within 1 year [1]. Despite its importance, the pathophysiology is still poorly understood, early recognition and prediction can be difficult, and there are no drugs licensed for the treatment of delirium.

According to various delirium guidelines, the treatment of delirium must primarily consist of non-pharmacological interventions [3-6]. The focus should be on treating the presumed causes of delirium as well as providing a suitable care environment. Pharmacological interventions with antipsychotics should only be considered in cases of intractable distress or when patients become a risk to themselves or others. Nonetheless, the place of antipsychotics in the treatment of delirium remains a topic of debate, and there are still many professionals who believe that antipsychotics can positively modify the course and outcomes of delirium 


\section{Key Points}

Delirium is associated with poor clinical outcomes.

Patients with delirium treated with antipsychotics (+ lorazepam) have a worse prognosis.

The findings of this study suggest caution when prescribing antipsychotics and lorazepam in older patients with delirium.

[7-9]. Moreover, in clinical practice, the benzodiazepine lorazepam is frequently added to antipsychotics to manage symptoms of delirium, despite that guidelines are very conservative on this topic $[3,10]$. This intervention is even more controversial, since there are studies suggesting that benzodiazepines may actually contribute to the development of delirium $[11,12]$.

We therefore conducted a real-life data study to investigate whether the use of antipsychotics, with or without lorazepam, increased the risk of prolonged hospital stay, post-discharge institutionalization, and in-hospital mortality in older patients with delirium.

\section{Methods}

\subsection{Study Design and Participants}

The present study was performed within a retrospective chart review study in which the association between anticholinergic drug burden and delirium, length of hospital stay (LOS), post-discharge institutionalization, and inhospital mortality were investigated. In the previous study, acutely ill patients aged 65 years and older admitted to the geriatric ward of the Erasmus University Medical Center, Rotterdam, The Netherlands, between 2012 and 2015 were included. Patients were excluded if they were hospitalized for $<3$ days or if data regarding drug use or outcome measures were not available [13]. In the present study, we included all patients who were diagnosed with delirium within the first 2 days of admission.

This study was conducted in accordance with the principles expressed in the Declaration of Helsinki. In The Netherlands, ethical approval and patient consent are not required for retrospective chart review studies in which data collected during routine clinical care are extracted and analyzed anonymously.

\subsection{Demographic and Clinical Data}

All data were collected from the medical records and included age, sex, prescribed drug use at the time of admission, cognitive performance prior to admission (no cognitive impairment, cognitive impairment/no dementia, and dementia), cause(s) of delirium, and the severity of comorbidities calculated using the Charlson Comorbidity Index (CCI) [14]. The CCI encompasses 19 medical conditions weighted with a score of $1-6$, with total scores ranging from 0 to 37 , and with higher scores indicating a more severe burden of comorbidities. Cognitive impairment and dementia were considered to be present when documented in the medical record. The anticholinergic drug burden score on admission was calculated using the Anticholinergic Risk Scale (ARS) [15]. On the ARS, anticholinergic drugs are ranked based on their potential to cause central and peripheral anticholinergic adverse effects (score range $0-3$ ). Drugs with a score of 1 have moderate anticholinergic potential and drugs with a score of 2 and 3 have a strong and very strong potential, respectively. The anticholinergic drug burden is the sum of scores assigned to each drug a patient is taking [15]. In addition, laboratory data and vital signs, obtained within the first 2 days of admission, were collected to calculate the burden of illness using the Laboratory Decision Tree Early Warning Score (LDT-EWS) [16] and the Modified Early Warning System (MEWS) [17]. The LDT-EWS combines seven laboratory test results: hemoglobin, white blood cell count, urea, creatinine, sodium, potassium, and albumin. The MEWS is based on five vital signs: systolic blood pressure, heart rate, respiratory rate, temperature, and the level of consciousness (the latter is measured using the AVPU score in which the A represents 'alert', V represents 'reacting to vocal stimuli', P represents 'reacting to pain', and U represents 'unresponsive'). A score is attributed to each observation based on the degree of physiological abnormality. These scores are summed to provide a total LDT-EWS or MEWS, and the total score represents the clinical status of the patient, with a higher score indicating a higher burden of illness [16, 17]. When the laboratory markers and vital signs were measured more than once within the first 2 days of admission, the first values were used. No data imputation was performed when more than $10 \%$ of a variable was missing.

\subsubsection{Definition of Delirium}

In our previous study, reported diagnoses of delirium were extracted from medical records by chart review [13]. On the geriatrics ward, a diagnosis of delirium is made by geriatricians as part of daily clinical practice, according to the Diagnostic and Statistical Manual of Mental Disorders 
(DSM), 4th and 5th editions criteria [18, 19], and is based on daily psychiatric examination, medical and nursing notes, the Delirium Observation Screening Scale (DOSS) scores [20], and information given by the patient's closest relative. Established diagnoses of delirium are reported in the medical records.

\subsubsection{Exposure to Antipsychotic Drugs and/or Lorazepam}

During the hospital stay, prescription data of antipsychotic drugs (i.e. haloperidol, quetiapine, clozapine, olanzapine, and risperidone) and lorazepam for the management of delirium were extracted from the medical records. Patients were stratified into three treatment groups based on whether or not they received antipsychotics and lorazepam to manage delirium: (0) no antipsychotics; (1) antipsychotics only; and (2) both antipsychotics and lorazepam. Prescribing of antipsychotics and lorazepam was physician-driven. All patients received non-pharmacological interventions.

\subsubsection{Outcome Measures}

The outcomes of interest were LOS, post-discharge institutionalization, and in-hospital mortality. LOS was defined as the number of days a patient was hospitalized, with the first day of admission being day 1 , while post-discharge institutionalization was defined as discharge to an institutional care facility rather than discharge to home.

\subsection{Statistical Analyses}

Differences in baseline characteristics between the three treatment groups were compared using the Chi-square test for normally distributed categorical variables, the Fisher-Freeman-Halton test for non-normally distributed categorical variables, analysis of variance (ANOVA) for normally distributed continuous variables, and the Kruskal-Wallis test for non-normally distributed continuous variables.

Logistic regression analysis was performed to calculate odds ratios (ORs) and corresponding 95\% confidence intervals (CIs) for LOS, post-discharge institutionalization, and in-hospital mortality (dependent variables) according to the antipsychotic treatment groups (treated as a continuous variable). LOS was divided into two groups based on the median value found in the overall sample of patients with delirium (11.0 days). Patients who died during the hospital stay were not included in analyses of LOS. Patients who resided in an institutional care facility before admission and patients who died during the hospital stay were not included in analyses with post-discharge institutionalization as an outcome. All patients were included in the analyses with regard to in-hospital mortality. All logistic regression analyses were adjusted for age, sex, and CCI.

The Statistical Package for the Social Sciences, version 25.0 (IBM Corporation, Armonk, NY, USA) was used to perform the statistical analyses. Results were considered statistically significant at a two-sided $p$ value of $<0.05$. Figures were constructed using GraphPad Prism 5.01 for Windows (GraphPad Software, San Diego, CA, USA).

\section{Results}

Of the 905 patients enrolled in this chart review study, 215 were diagnosed with delirium within the first 2 days of the hospital stay; three of these 215 patients received lorazepam without an antipsychotic and were subsequently excluded. In total, 212 patients were included in the present study; 40 (18.9\%) patients did not receive an antipsychotic, $123(58.0 \%)$ received antipsychotics only, and 49 (23.1\%) patients received antipsychotics and lorazepam. The mean age of the overall sample was $81.9 \pm 5.6$ years (range $65-101)$ and $56 \%$ were male. In total, $32.5 \%$ of patients had no cognitive impairment, $42.0 \%$ had cognitive impairment not (yet) diagnosed as dementia, and $25.5 \%$ had dementia prior to admission. Table 1 presents the patient demographic and clinical characteristics. No statistically significant differences in baseline characteristics were found among the groups, as well as in the inflammatory markers, i.e. C-reactive protein and white blood cell count, and burden of illness score measured using the LDT-EWS. However, a statistically significant difference was found in the median MEWS score, with the lowest median MEWS score in patients who received antipsychotics and lorazepam $(p=0.007)$. Furthermore, patients who received antipsychotics and lorazepam seemed to be slightly more dependent than patients in the other two groups, since they resided more often in an institutional care facility prior to admission, but the difference did not reach statistical significance.

Table 2 presents the causes of delirium. Urinary retention and dehydration seemed to more often be the precipitating cause in patients who received antipsychotics and lorazepam, whereas electrolyte disturbances were more often the precipitating cause in patients who did not receive antipsychotics compared with the other groups (no significance tests were performed due to the number of comparisons).

\subsection{Use of Antipsychotics}

Table 3 presents the prescription data of antipsychotics during delirium. The most prescribed antipsychotic was haloperidol, followed by quetiapine. There were no differences in the distribution of antipsychotics among the two groups 
Table 1 Baseline characteristics of the study participants

\begin{tabular}{|c|c|c|c|c|}
\hline Variable & No antipsychotics $[n=40]$ & Antipsychotics only [ $n=123]$ & $\begin{array}{l}\text { Antipsychotics }+ \\
\text { lorazepam }[n=49]\end{array}$ & $p$ value \\
\hline Age, years & $79.9 \pm 6.9$ & $82.2 \pm 6.6$ & $82.7 \pm 6.6$ & $0.109^{\mathrm{a}}$ \\
\hline Male $(\%)$ & 55.0 & 57.7 & 53.1 & $0.846^{\mathrm{b}}$ \\
\hline Place of residence before admission (\%) & & & & $0.839^{\mathrm{b}}$ \\
\hline Home (with or without home care) & 75.0 & 72.4 & 69.4 & \\
\hline Institutional care facility & 25.0 & 27.6 & 30.6 & \\
\hline Cognition $(\%)$ & & & & $0.119^{\mathrm{b}}$ \\
\hline No cognitive impairment & 25.0 & 39.8 & 20.4 & \\
\hline Cognitive impairment (no dementia) & 47.5 & 37.4 & 49.0 & \\
\hline Dementia & 27.5 & 22.8 & 30.6 & \\
\hline Charlson Comorbidity Index & $3.0(1.0-4.0)$ & $3.0(2.0-4.0)$ & $2.0(1.0-4.0)$ & $0.262^{c}$ \\
\hline Number of prescribed drugs & $7.5(6.0-12.5)$ & $9.0(6.0-13.0)$ & $7.0(5.0-9.5)$ & $0.061^{\mathrm{c}}$ \\
\hline ARS score & $0.0(0.0-1.0)$ & $0.0(0.0-1.0)$ & $0.0(0.0-1.0)$ & $0.543^{\mathrm{c}}$ \\
\hline \multicolumn{5}{|l|}{ Laboratory markers } \\
\hline C-reactive protein $(\mathrm{mg} / \mathrm{L})$ & $36.8(14.0-104.0)$ & $47.3(13.18-130.75)$ & $43.0(13.5-107.5)$ & $0.906^{\mathrm{c}}$ \\
\hline eGFR (mL/min) & $48.0(30.5-64.5)$ & $54.0(37.0-79.0)$ & $55.0(35.5-78.0)$ & $0.223^{\mathrm{c}}$ \\
\hline Hemoglobin (g/dL) & $12.7 \pm 2.04$ & $12.5 \pm 1.98$ & $12.6 \pm 1.71$ & $0.884^{\mathrm{a}}$ \\
\hline White blood cell count $\left(10^{9} / \mathrm{L}\right)$ & $10.95(8.6-15.27)$ & $10.76(7.98-13.95)$ & $9.60(7.8-13.0)$ & $0.399^{c}$ \\
\hline Urea $(\mathrm{mmol} / \mathrm{L})$ & $9.8(6.28-17.5)$ & $9.6(6.75-15.23)$ & $9.6(6.5-15.1)$ & $0.908^{\mathrm{c}}$ \\
\hline Creatinine $(\mu \mathrm{mol} / \mathrm{L})$ & $109.0(81.0-136.0)$ & $103.0(72.75-142.5)$ & $94.0(68.5-158.0)$ & $0.382^{c}$ \\
\hline Sodium $(\mathrm{mmol} / \mathrm{L})$ & $138.0(136.0-141.0)$ & $138.0(135.0-141.0)$ & $138.0(134.5-140.0)$ & $0.877^{\mathrm{c}}$ \\
\hline Potassium (mmol/L) & $4.2(3.7-4.8)$ & $4.4(3.9-4.7)$ & $4.4(4.2-4.9)$ & $0.169^{c}$ \\
\hline Albumin $(\mathrm{g} / \mathrm{L})$ & $35.0(30.8-40.0)$ & $34.0(31.0-38.0)$ & $36.0(33.0-39.3)$ & $0.406^{\mathrm{c}, \mathrm{d}}$ \\
\hline LDT-EWS & $5.0(3.0-9.0)$ & $5.0(2.0-8.0)$ & $4.0(2.5-8.0)$ & $0.643^{\mathrm{c}, \mathrm{d}}$ \\
\hline \multicolumn{5}{|l|}{ Vital signs } \\
\hline Systolic blood pressure, $\mathrm{mmHg}$ & $140.0(128.5-163.5)$ & $134.0(118.0-158.0)$ & $134.0(120.0-151.5)$ & $0.310^{\mathrm{c}}$ \\
\hline Heart rate, beats/min & $88.0(71.3-103.0)$ & $88.0(77.0-102.0)$ & $87.0(70.0-95.0)$ & $0.285^{c}$ \\
\hline Respiratory rate, breaths/min & $22.0(16.5-28.0)$ & $22.5(16.0-28.5)$ & $19.0(16.0-24.0)$ & $0.273^{\mathrm{c}, \mathrm{d}}$ \\
\hline Temperature $\left({ }^{\circ} \mathrm{C}\right)$ & $37.1(36.1-38.1)$ & $37.0(36.5-37.8)$ & $36.7(36.5-37.2)$ & $0.158^{c}$ \\
\hline AVPU score ${ }^{\mathrm{e}}$ & $0.0(0.0-1.0)$ & $0.0(0.0-1.0)$ & $0.0(0.0-1.0)$ & $0.556^{\mathrm{c}}$ \\
\hline MEWS & $3.0(1.5-4.0)$ & $3.0(2.0-5.0)$ & $2.0(1.0-3.0)$ & $0.007^{\mathrm{c}, \mathrm{d}}$ \\
\hline
\end{tabular}

Data are expressed as mean \pm SD for normally distributed continuous variables, median (interquartile range) for non-normally distributed continuous variables, and percentages for categorical variables

ANOVA analysis of variance, ARS Anticholinergic Risk Scale, AVPU Alert Verbal Pain Unresponsive, $e G F R$ estimated glomerular filtration rate, LDT-EWS Laboratory Decision Tree Early Warning Score, MEWS Modified Early Warning System, SD standard deviation

${ }^{\mathrm{a}} \mathrm{ANOVA}$

${ }^{\mathrm{b}}$ Chi-square test

${ }^{c}$ Kruskal-Wallis test

${ }^{\mathrm{d}}$ More than $10 \%$ missing data: albumin was available for 131 patients $(26,75,30$ patients, respectively); LDT-EWS was available for 128 patients $(26,73,29$ patients, respectively); respiratory rate was available for 130 patients $(29,78,23$ patients, respectively); MEWS was available for 129 patients $(29,77,23$ patients, respectively)

'Scoring: 0 for 'alert'; 1 for 'reacting to vocal stimuli'; 2 for 'reacting to pain'; 3 for 'unresponsive'

who received antipsychotics. In the group who received antipsychotics and lorazepam, statistically more patients were prescribed two antipsychotics than in the group who received antipsychotics only ( $22.4 \%$ vs. $6.5 \%, p=0.003)$. Furthermore, patients who were treated with antipsychotics and lorazepam more often received antipsychotic drug prescriptions on a regular basis (with or without 'as needed' antipsychotics; $p=0.037$ ) and were treated statistically significantly longer with antipsychotics (median 11.0 days, interquartile range [IQR] 7.0-16.5) than patients who received antipsychotics only (median 7.0, IQR 4.0-10.0; $p$ $<0.001)$. 
Table 2 Causes of delirium

\begin{tabular}{|c|c|c|c|c|}
\hline & Causes of delirium & $\begin{array}{l}\text { No antipsychotics } \\
{[n=40], \%^{\mathrm{a}}}\end{array}$ & $\begin{array}{l}\text { Antipsychotics only } \\
{[n=123], \%^{\mathrm{a}}}\end{array}$ & $\begin{array}{l}\text { Antipsychotics }+ \\
\text { lorazepam }[n=49] \\
\%^{\mathrm{a}}\end{array}$ \\
\hline \multirow[t]{12}{*}{ Precipitating factors } & Infection & 60.0 & 73.2 & 71.4 \\
\hline & Electrolyte disturbance & 25.0 & 9.8 & 16.3 \\
\hline & Medication & 15.0 & 13.0 & 16.3 \\
\hline & Metabolic disorder & 5.0 & 7.3 & 4.1 \\
\hline & Dehydration & 5.0 & 3.3 & 10.2 \\
\hline & Urinary retention & 0.0 & 3.3 & 14.3 \\
\hline & Desaturation/hypoxia & 2.5 & 4.1 & 0.0 \\
\hline & Hypothermia or fever & 2.5 & 2.4 & 0.0 \\
\hline & Hematoma & 0.0 & 2.4 & 4.1 \\
\hline & Rhabdomyolysis & 0.0 & 0.0 & 4.1 \\
\hline & Fall & 0.0 & 1.6 & 2.0 \\
\hline & Surgery & 0.0 & 0.8 & 2.0 \\
\hline \multirow{2}{*}{$\begin{array}{l}\text { Predisposing or precipitating } \\
\text { factor }\end{array}$} & Renal failure (chronic and acute) & 10.0 & 10.6 & 12.2 \\
\hline & Heart failure (chronic and acute) & 0.0 & 6.5 & 8.2 \\
\hline
\end{tabular}

${ }^{\text {a }}$ The total percentage does not add up to 100 because delirium is often a multifactorial condition

Table 3 Prescription data of antipsychotics

$\begin{array}{ll}\text { Antipsychotics only } & \text { Antipsychotics + lorazepam } \\ {[n=123]} & {[n=49]}\end{array}$

Antipsychotics during delirium (\%)

\begin{tabular}{|c|c|c|c|}
\hline Haloperidol & 88.6 & 89.8 & $0.824^{\mathrm{a}}$ \\
\hline Quetiapine & 13.0 & 24.5 & $0.066^{\mathrm{a}}$ \\
\hline Clozapine & 4.9 & 6.1 & $0.715^{\mathrm{b}}$ \\
\hline Risperidone & - & 2.0 & $0.285^{\mathrm{b}}$ \\
\hline Olanzapine & - & - & - \\
\hline Two antipsychotics $(\%)$ & 6.5 & 22.4 & $0.003^{\mathrm{a}}$ \\
\hline Basis of antipsychotic drug prescriptions (\%) & & & $0.037^{\mathrm{a}}$ \\
\hline PRN & 15.4 & 4.1 & \\
\hline Regular & 47.2 & 40.8 & \\
\hline Both $(\mathrm{PRN}+\text { regular })^{\mathrm{d}}$ & 37.4 & 55.1 & \\
\hline Number of hospital days on antipsychotics [median (IQR)] & $7.0(4.0-10.0)$ & $11.0(7.0-16.5)$ & $<0.001^{\mathrm{c}}$ \\
\hline \multicolumn{4}{|l|}{ Basis of lorazepam prescriptions (\%) } \\
\hline PRN & - & 38.8 & - \\
\hline Regular & - & 30.6 & - \\
\hline Both $(\mathrm{PRN}+\text { regular })^{\mathrm{d}}$ & - & 30.6 & - \\
\hline Number of hospital days on lorazepam [median (IQR)] & - & $7.0(3.0-11.5)$ & - \\
\hline
\end{tabular}

$I Q R$ interquartile range, $P R N$ pro re nata (as needed)

${ }^{\mathrm{a}} \mathrm{Chi}$-square test

${ }^{\mathrm{b}}$ Fisher's exact test

${ }^{\mathrm{c}}$ Mann-Whitney $U$ test

${ }^{\mathrm{d}}$ Contains both simultaneous and consecutive prescriptions 


\subsection{Clinical Outcomes}

In unadjusted models, patients who received antipsychotics and lorazepam had a longer median LOS (median 12.0 days, IQR 9.0-17.5) than those who received antipsychotics only (median 10.0, IQR 7.0-14.0) and those who did not receive antipsychotics (median 8.0, IQR 5.3-10.8; $p<0.001$ ) (Fig. 1a). Furthermore, the incidence of post-discharge institutionalization was higher in patients who received antipsychotics and lorazepam (81.6\%) than in those who received antipsychotics only (59.3\%) and those who did not receive antipsychotics $(45.0 \% ; p<0.001)$ (Fig. 1b). No statistically significant difference was found in in-hospital mortality; however, a trend was seen among the groups, with the highest mortality rate in patients who received antipsychotics and lorazepam ( $p=0.392)$ (Fig. 1c).

Table 4 presents the adjusted ORs and corresponding 95\% CIs for LOS $\geq 11$ days, post-discharge institutionalization, and in-hospital mortality based on the antipsychotic treatment groups. Each change in treatment (from no antipsychotics to antipsychotics, and from antipsychotics to antipsychotics plus lorazepam) was associated with a 2.47 -fold higher odds of having a prolonged LOS (OR 2.47, 95\% CI 1.51-4.07) and a 4.47-fold higher odds of being institutionalized after discharge (OR 4.47, 95\% CI 2.27-8.79). No association was found between the antipsychotic treatment groups and in-hospital mortality (OR 1.57, 95\% CI $0.76-3.25)$.

\section{Discussion}

In the present study, we found that older patients with delirium who are treated with antipsychotics have a worse prognosis, and patients who additionally receive lorazepam have even poorer clinical outcomes.

To the best of our knowledge, this is the first study in which clinical outcomes of delirium are compared in different treatment groups within a real-life sample of acutely ill older patients. Weaver et al. and Daniels et al. compared outcomes in critically ill patients with delirium and found that patients who were treated with antipsychotics had a longer duration of delirium and a longer intensive care unit (ICU) and hospital stay than those who did not receive antipsychotics [21, 22]. In contrast to our study, Weaver et al. found no difference in discharge disposition and in-hospital mortality; however, the patients were considerably younger, and therefore perhaps less frail, than the patients included in our study [21]. Nguyen et al. investigated the pharmacological management of delirium among older hospitalized patients and observed an increased mortality rate in patients who received a benzodiazepine during their delirium episode [23]. It is unclear which benzodiazepines were used
A

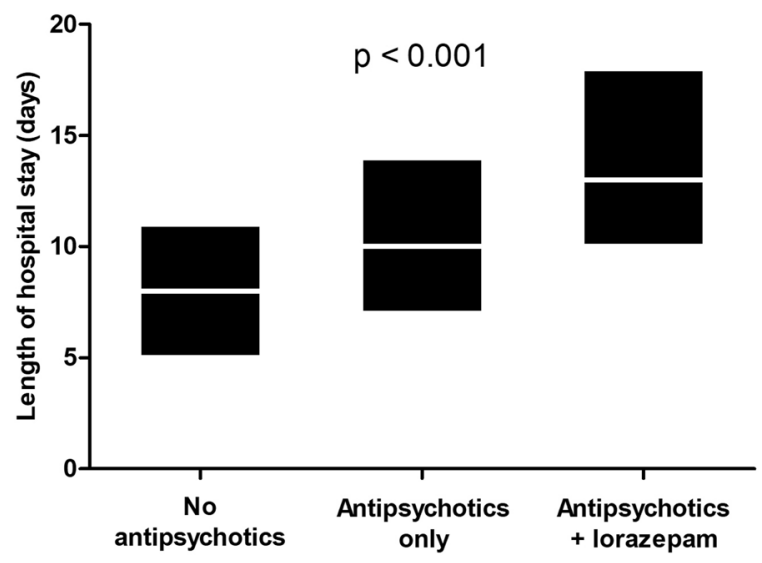

B

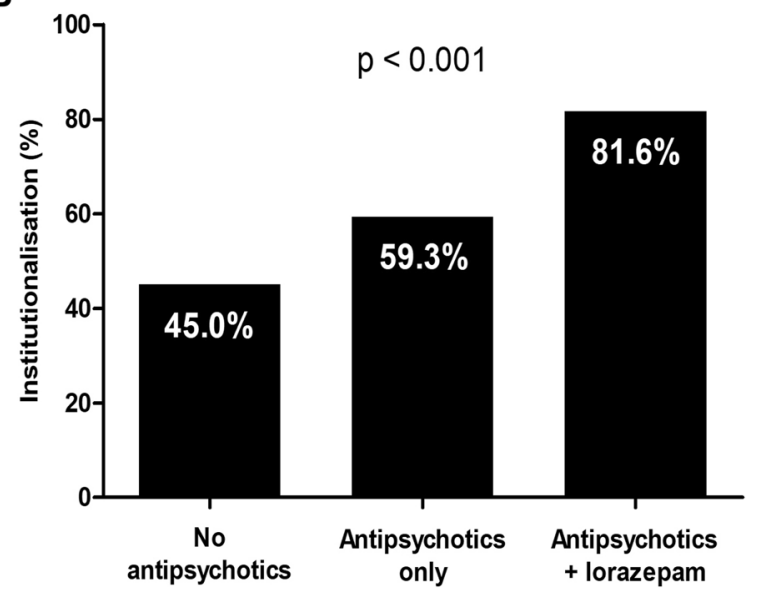

C

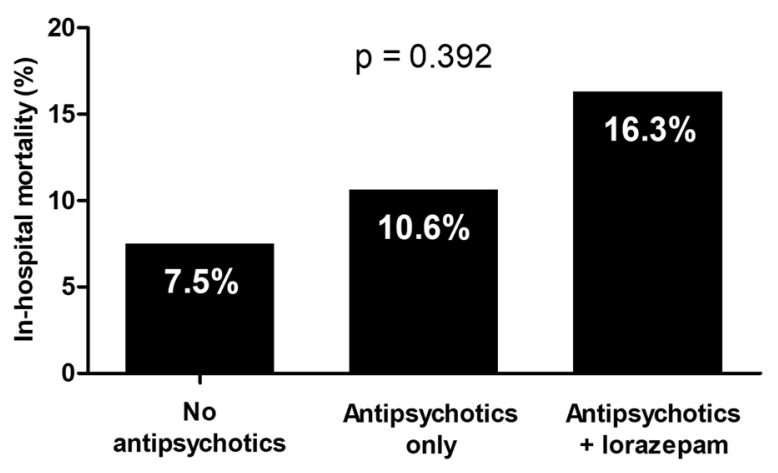

Fig. 1 a Length of hospital stay, b post-discharge institutionalization, and $\mathbf{c}$ in-hospital mortality in the three treatment groups. Length of hospital stay is presented as median (interquartile range)

and whether the indication was delirium, but this finding highlights that benzodiazepines during delirium might be harmful.

The findings of the present retrospective chart review study are not in line with the findings of systematic reviews and meta-analyses, which reported no differences in several 
Table 4 Odds ratios for clinical outcomes based on the antipsychotic treatment groups

\begin{tabular}{|c|c|c|c|}
\hline & $\begin{array}{l}\text { LOS } \geq 11 \text { days } \\
\text { aOR }(95 \% \mathrm{CI})\end{array}$ & $\begin{array}{l}\text { Post-discharge institutionalization } \\
\text { aOR }(95 \% \mathrm{CI})\end{array}$ & $\begin{array}{l}\text { In-hospital mortality } \\
\text { aOR }(95 \% \mathrm{CI})\end{array}$ \\
\hline Antipsychotic treatment group ${ }^{a}$ & $2.47(1.51-4.07)^{b}$ & $4.47(2.27-8.79)^{\mathrm{c}}$ & $1.57(0.76-3.25)$ \\
\hline
\end{tabular}

$a O R$ adjusted odds ratio, $C I$ confidence interval, $L O S$ length of hospital stay

Bolded values indicate statistical significance

${ }^{\text {a }}$ The antipsychotic treatment groups are defined as $0=$ no antipsychotics, $1=$ antipsychotics only, and $2=$ antipsychotics + lorazepam. Models are adjusted for age, sex and Charlson Comorbidity Index

${ }^{\mathrm{b}}$ Patients who died during the hospital stay were excluded

${ }^{c}$ Patients who resided in an institutional care facility before admission, as well as patients who died during the hospital stay, were excluded

clinical outcomes, including LOS, delirium duration and severity, and mortality in patients with delirium who received antipsychotics or placebo [24, 25]. These reviews included studies performed in various populations, including ICU patients. A Cochrane review of Burry et al., which focused specifically on the efficacy of antipsychotics in the treatment of delirium in hospitalized non-ICU patients, found that antipsychotics did not reduce delirium severity or alter mortality. None of the included studies in that review reported on delirium duration, hospital LOS, or discharge disposition [26]. On the other hand, a randomized controlled trial (RCT) performed in palliative care patients reported that patients who received oral haloperidol or oral risperidone for delirium had worse delirium symptoms and died sooner than patients who received an oral placebo [27]. Furthermore, lorazepam is frequently used in clinical practice, but the number of RCTs that have investigated the effect of lorazepam on delirium and outcomes is scarce. A Cochrane review identified two small RCTs in non-ICU settings, but there was insufficient evidence to support or reject the use of benzodiazepines during delirium [28].

It might be possible that our findings are confounded by indication, for example by the duration and severity of delirium. Previous studies performed in older patients admitted to a general medicine ward and older patients who underwent acute hip fracture surgery have reported that the 3 - and 6-month mortality risk increases with each day that delirium persists [29, 30]. In the present study, delirium duration was not measured since most patients already had delirium on admission. Considering that pharmacological interventions are likely to be initiated at a later stage (when a patient does not respond to non-pharmacological interventions and is distressed or is a risk to self or others), it might be possible that patients who were prescribed antipsychotics had a longer duration of delirium. Moreover, the severity of delirium might have influenced the results. Marcantonio et al. found that patients with severe delirium were three times more likely to be institutionalized or dead after 6 months [31]. Tropea et al. investigated the pharmacological management of delirium in patients admitted to a general medical or orthopedic hospital unit and found that patients with severe symptoms of delirium were more often prescribed antipsychotics and benzodiazepines, had a significantly higher median LOS, and were more often discharged to a geriatric medical evaluation unit, but did not have a statistically significantly higher in-hospital mortality risk than patients without severe symptoms [32]. In the present study, delirium severity was not measured with a tool during routine clinical practice and the DOSS was not consistently rated as a proxy for delirium severity [33]. However, we found that $22 \%$ of the patients who were treated with antipsychotics and lorazepam received two antipsychotics during the hospital stay, were treated with antipsychotics for more days, and more often received antipsychotics on a regular basis. This might indicate that one antipsychotic drug was ineffective or insufficient and that the delirium was severe and persisted for a few days.

It seems unlikely that our findings are confounded by the severity of the underlying acute illness, since we did not find any differences in the levels of several laboratory markers or in the burden of illness score measured using the LDTEWS. Only the MEWS was significantly different among the groups, with the lowest MEWS score in the group who received antipsychotics and lorazepam. This finding suggests that this latter group had the least abnormalities in vital signs. Weaver et al. used the Simplified Acute Physiology Score (SAPS) III to measure the burden of illness and, comparable with our findings, found no difference between patients who did or did not use antipsychotics [21]. Nonetheless, we cannot exclude that the severity of the underlying illness increased during the hospital stay.

It might also be speculated that the high prevalence of cognitive impairment and dementia has contributed to worse outcomes among the groups. Several studies have found that patients with delirium superimposed on dementia have higher risks of institutionalization and mortality than patients with delirium alone [34-36]. However, in the present study, we did not find a statistically significant difference in the prevalence of cognitive impairment and dementia among the groups, and therefore it seems unlikely that our 
findings are confounded by the patients' cognitive status. Moreover, it has been reported that antipsychotics are associated with increased mortality in patients with dementia [37]. When we stratified our data based on cognitive status, we observed a comparable positive trend in LOS and institutionalization among the groups, whereas mortality decreased in patients with dementia, with the lowest mortality rate in patients who received antipsychotics and lorazepam (data not shown). These findings suggest that an interaction between dementia and antipsychotics was absent.

It might also be speculated that adverse effects caused by the pharmacological interventions may have influenced the results. Depending on the affinities for various receptor systems, including dopaminergic, serotonergic, muscarinic, adrenergic, and histaminergic receptors, antipsychotics may cause a wide range of adverse effects, such as extrapyramidal symptoms, QTc prolongation, sedation, and metabolic derangements such as hyperglycemia [38]. From systematic reviews, there is little evidence that antipsychotics are associated with harmful neurologic and cardiac effects in patients with delirium [24, 26]. However, many RCTs have excluded patients with neurologic and cardiovascular diseases or other risk factors, which can underestimate the adverse effects in daily clinical practice [24]. In our study, we found that the group who received antipsychotics and lorazepam had the lowest MEWS. It might be possible that these patients did not have many abnormalities in vital signs on admission to hospital, but it might also be possible that these patients had a decreased ability to correct abnormalities. In that case, antipsychotics and lorazepam may worsen the clinical situation, for example due to their effects on the respiratory and cardiovascular systems [38-40]. In addition, we found that patients who were treated with antipsychotics and lorazepam more frequently received antipsychotic drug prescriptions on a regular basis and were treated statistically significantly longer with antipsychotics than patients who received antipsychotics only. Therefore, it might be possible that patients who received antipsychotics plus lorazepam were exposed to an increased dose of antipsychotics. Maust et al. found that patients with dementia who were exposed to a highdose haloperidol-equivalent ( $>3 \mathrm{mg} /$ day) had an increased mortality risk compared with patients who received a lowdose haloperidol-equivalent ( $<1.5 \mathrm{mg} /$ day) [37]. Moreover, a review on antipsychotic drug use in delirium concluded that high doses of haloperidol ( $>4.5 \mathrm{mg} /$ day) are associated with increased incidences of adverse effects [41]. These findings suggest that a dose-response relationship may be an explanation for our findings.

\subsection{Limitations}

This study has several limitations. First, the use and choice of antipsychotics during delirium differs among centers and countries, therefore the single-center nature of this study limits the generalizability of the findings. Second, delirium severity was not measured. It might be possible that patients with more severe delirium were more likely to be prescribed antipsychotics and/or lorazepam. Third, the degree to which the underlying cause(s) of delirium were successfully reversed is unknown. Patients in whom the underlying cause(s) were difficult to treat might have had a longer delirium and poorer outcomes. Furthermore, other pre-existing risk factors, not assessed in this study, might have influenced our findings. Fourth, delirium subtype was not recorded. Subtype influences the way pharmacological interventions are used, with patients with hyperactive delirium usually receiving more antipsychotics with or without lorazepam. Previous studies have reported conflicting findings, but the hypoactive subtype is described more commonly as the subtype with the worst prognosis. Therefore, we believe that our findings are not, or are minimally, influenced by delirium subtype [42-44]. Fifth, daily drug dose and dosing schedule of the pharmacological interventions were not collected, therefore we cannot examine dose-response associations. Knowing the exact doses would have allowed us to more precisely assess the real response to drugs. Moreover, we cannot confirm that patients with 'as needed' prescriptions for antipsychotics and lorazepam also received these drugs during the hospital stay, and this may have led to an overestimation of the treatment duration. Lastly, we investigated only short-term outcomes. The possible association between antipsychotics, lorazepam, and long-term outcomes needs further investigation.

This study also has some strengths. First, patients were intensively monitored for clinical symptoms of delirium and a diagnosis of delirium was made by a geriatrician using the DSM criteria. This makes it less likely that delirium was missed or that symptoms were misdiagnosed. Second, the number of studies that have investigated the effect of lorazepam during delirium, especially in acutely ill older patients, is scarce and therefore the present study adds new knowledge.

\section{Conclusion}

This study shows that older patients with delirium who are prescribed antipsychotics, with or without lorazepam, have poorer outcomes than patients who are not treated with antipsychotics. These findings suggest that clinicians should be cautious about routine prescribing of these drugs to older patients with delirium. Further investigation is needed to clarify this association. Until the exact relationship between antipsychotics, lorazepam, and poor outcomes in delirium is clear, clinicians should use these drugs with caution and be aware of the lack of evidence supporting their efficacy and tolerability in older patients. 
Acknowledgements None.

\section{Declarations}

Funding No sources of funding were used for the conduct of this study or the preparation of this article.

Conflicts of Interest Angelique Egberts, Hava Alan, Gijsbertus Ziere and Francesco U.S. Mattace-Raso declare they have no conflicts of interest.

Ethical approval/Informed consent In The Netherlands, ethical approval or patient consent are not required for retrospective chart review studies in which data collected during routine clinical care are extracted and analyzed anonymously.

Consent for publication Not applicable.

Availability of data and material The datasets generated and/or analyzed during the current study are available from the corresponding author upon reasonable request.

\section{Code availability Not applicable.}

Author contributions AE, HA, GZ, FMR: Study concept and design. AE, HA: Acquisition of data. AE, HA, GZ, FMR: Analysis and interpretation of data. AE: Drafting of the manuscript. HA, GZ, FMR: Critical revision of the manuscript for important intellectual content. All authors approved the final version of the manuscript.

Open Access This article is licensed under a Creative Commons Attribution-NonCommercial 4.0 International License, which permits any non-commercial use, sharing, adaptation, distribution and reproduction in any medium or format, as long as you give appropriate credit to the original author(s) and the source, provide a link to the Creative Commons licence, and indicate if changes were made. The images or other third party material in this article are included in the article's Creative Commons licence, unless indicated otherwise in a credit line to the material. If material is not included in the article's Creative Commons licence and your intended use is not permitted by statutory regulation or exceeds the permitted use, you will need to obtain permission directly from the copyright holder. To view a copy of this licence, visit http://creativecommons.org/licenses/by-nc/4.0/.

\section{References}

1. Witlox J, Eurelings LS, de Jonghe JF, Kalisvaart KJ, Eikelenboom P, van Gool WA. Delirium in elderly patients and the risk of postdischarge mortality, institutionalization, and dementia: a meta-analysis. JAMA. 2010;304:443-51.

2. Siddiqi N, House AO, Holmes JD. Occurrence and outcome of delirium in medical in-patients: a systematic literature review. Age Ageing. 2006;35:350-64.

3. Scottish Intercollegiate Guidelines Network. Risk reduction and management of delirium. 2019. https://www.sign.ac.uk/sign157-delirium.html. Accessed 25 Mar 2020.

4. National Institute for Health and Care Excellence (NICE). Delirium: Diagnosis, prevention and management. Clinical Guideline 103. 2010, update March 2019. www.nice.org.uk/CG103. Accessed 25 Mar 2020.
5. American Geriatrics Society. Clinical Practice Guideline for Postoperative Delirium in Older Adults. 2014. https://geriatricscareo nline.org/. Accessed 25 Mar 2020.

6. Dutch Geriatrics Society [Nederlandse Vereniging voor Klinische Geriatrie]. Delier bij volwassenen [in Dutch]. 2013. https://richt lijnendatabase.nl/. Accessed 25 Mar 2020.

7. Meagher D, Agar MR, Teodorczuk A. Debate article: Antipsychotic medications are clinically useful for the treatment of delirium. Int J Geriatr Psychiatry. 2018;33:1420-7.

8. Morandi A, Davis D, Taylor JK, et al. Consensus and variations in opinions on delirium care: a survey of European delirium specialists. Int Psychogeriatr. 2013;25:2067-75.

9. Bellelli G, Morandi A, Zanetti E, et al. Recognition and management of delirium among doctors, nurses, physiotherapists, and psychologists: an Italian survey. Int Psychogeriatr. 2014;26:2093-102.

10. Carnes M, Howell T, Rosenberg M, Francis J, Hildebrand C, Knuppel J. Physicians vary in approaches to the clinical management of delirium. J Am Geriatr Soc. 2003;51:234-9.

11. Pandharipande P, Shintani A, Peterson J, et al. Lorazepam is an independent risk factor for transitioning to delirium in intensive care unit patients. Anesthesiology. 2006;104:21-6.

12. Zaal IJ, Devlin JW, Hazelbag M, et al. Benzodiazepine-associated delirium in critically ill adults. Intensive Care Med. 2015;41:2130-7.

13. Egberts A, van der Craats ST, van Wijk MD, Alkilabe S, van den Bemt P, Mattace-Raso FUS. Anticholinergic drug exposure is associated with delirium and postdischarge institutionalization in acutely ill hospitalized older patients. Pharmacol Res Perspect. 2017;5:e00310.

14. Charlson ME, Pompei P, Ales KL, MacKenzie CR. A new method of classifying prognostic comorbidity in longitudinal studies: development and validation. J Chronic Dis. 1987;40:373-83.

15. Rudolph JL, Salow MJ, Angelini MC, McGlinchey RE. The anticholinergic risk scale and anticholinergic adverse effects in older persons. Arch Intern Med. 2008;168:508-13.

16. Jarvis SW, Kovacs C, Badriyah T, et al. Development and validation of a decision tree early warning score based on routine laboratory test results for the discrimination of hospital mortality in emergency medical admissions. Resuscitation. 2013;84:1494-9.

17. Subbe CP, Kruger M, Rutherford P, Gemmel L. Validation of a modified Early Warning Score in medical admissions. QJM. 2001;94:521-6.

18. American Psychiatric Association. Diagnostic and statistical manual of mental disorders. 4th ed. Washington, DC: American Psychiatric Association; 2000. ((Text revision)).

19. American Psychiatric Association. Diagnostic and statistical manual of mental disorders. 5th ed. Arlington: American Psychiatric Association; 2013.

20. Schuurmans MJ, Shortridge-Baggett LM, Duursma SA. The Delirium Observation Screening Scale: a screening instrument for delirium. Res Theory Nurs Pract. 2003;17:31-50.

21. Weaver CB, Kane-Gill SL, Gunn SR, Kirisci L, Smithburger PL. A retrospective analysis of the effectiveness of antipsychotics in the treatment of ICU delirium. J Crit Care. 2017;41:234-9.

22. Daniels LM, Nelson SB, Frank RD, Park JG. Pharmacologic treatment of intensive care unit delirium and the impact on duration of delirium, length of intensive care unit stay, length of hospitalization, and 28-day mortality. Mayo Clin Proc. 2018;93:1739-48.

23. Nguyen P, Malachane A, Vu T. Antipsychotic prescription patterns in the management of delirium symptoms in hospitalized elderly patients. Proc Singap Healthc. 2017;26:230-4.

24. Nikooie R, Neufeld KJ, Oh ES, et al. Antipsychotics for treating delirium in hospitalized adults: a systematic review. Ann Intern Med. 2019;171:485-95. 
25. Neufeld KJ, Yue J, Robinson TN, Inouye SK, Needham DM. Antipsychotic medication for prevention and treatment of delirium in hospitalized adults: a systematic review and meta-analysis. J Am Geriatr Soc. 2016;64:705-14.

26. Burry L, Mehta S, Perreault MM, et al. Antipsychotics for treatment of delirium in hospitalised non-ICU patients. Cochrane Database Syst Rev. 2018;6:CD00594.

27. Agar MR, Lawlor PG, Quinn S, et al. Efficacy of oral risperidone, haloperidol, or placebo for symptoms of delirium among patients in palliative care: a Randomized Clinical Trial. JAMA Intern Med. 2017;177:34-42.

28. Li Y, Ma J, Jin Y, et al. Benzodiazepines for treatment of patients with delirium excluding those who are cared for in an intensive care unit. Cochrane Database Syst Rev. 2020;2:CD012670.

29. Gonzalez M, Martinez G, Calderon J, et al. Impact of delirium on short-term mortality in elderly inpatients: a prospective cohort study. Psychosomatics. 2009;50:234-8.

30. Bellelli G, Mazzola P, Morandi A, et al. Duration of postoperative delirium is an independent predictor of 6-month mortality in older adults after hip fracture. J Am Geriatr Soc. 2014;62:1335-40.

31. Marcantonio E, Ta T, Duthie E, Resnick NM. Delirium severity and psychomotor types: their relationship with outcomes after hip fracture repair. J Am Geriatr Soc. 2002;50:850-7.

32. Tropea J, Slee J, Holmes AC, Gorelik A, Brand CA. Use of antipsychotic medications for the management of delirium: an audit of current practice in the acute care setting. Int Psychogeriatr. 2009;21:172-9.

33. Scheffer AC, van Munster BC, Schuurmans MJ, de Rooij SE. Assessing severity of delirium by the Delirium Observation Screening Scale. Int J Geriatr Psychiatry. 2011;26:284-91.

34. Morandi A, Davis D, Fick DM, et al. Delirium superimposed on dementia strongly predicts worse outcomes in older rehabilitation inpatients. J Am Med Dir Assoc. 2014;15:349-54.
35. McCusker J, Cole M, Dendukuri N, Belzile E, Primeau F. Delirium in older medical inpatients and subsequent cognitive and functional status: a prospective study. CMAJ. 2001;165:575-83.

36. Bellelli G, Frisoni GB, Turco R, Lucchi E, Magnifico F, Trabucchi M. Delirium superimposed on dementia predicts 12-month survival in elderly patients discharged from a postacute rehabilitation facility. J Gerontol A Biol Sci Med Sci. 2007;62:1306-9.

37. Maust DT, Kim HM, Seyfried LS, et al. Antipsychotics, other psychotropics, and the risk of death in patients with dementia: number needed to harm. JAMA Psychiatry. 2015;72:438-45.

38. Solmi M, Murru A, Pacchiarotti I, et al. Safety, tolerability, and risks associated with first- and second-generation antipsychotics: a state-of-the-art clinical review. Ther Clin Risk Manag. 2017;13:757-77.

39. Wang MT, Tsai CL, Lin CW, Yeh CB, Wang YH, Lin HL. Association between antipsychotic agents and risk of acute respiratory failure in patients with chronic obstructive pulmonary disease. JAMA Psychiatry. 2017;74:252-60.

40. Barr J, Fraser GL, Puntillo K, et al. Clinical practice guidelines for the management of pain, agitation, and delirium in adult patients in the intensive care unit. Crit Care Med. 2013;41:263-306.

41. Lonergan E, Britton AM, Luxenberg J, Wyller T. Antipsychotics for delirium. Cochrane Database Syst Rev. 2007;2:CD005594.

42. Krewulak KD, Stelfox HT, Ely EW, Fiest KM. Risk factors and outcomes among delirium subtypes in adult ICUs: a systematic review. J Crit Care. 2020;56:257-64.

43. Bellelli G, Speciale S, Barisione E, Trabucchi M. Delirium subtypes and 1-year mortality among elderly patients discharged from a post-acute rehabilitation facility. J Gerontol A Biol Sci Med Sci. 2007;62:1182-3.

44. Jackson TA, Wilson D, Richardson S, Lord JM. Predicting outcome in older hospital patients with delirium: a systematic literature review. Int J Geriatr Psychiatry. 2016;31:392-9. 